\title{
THE VALUE OF OPHTHALMIC TREATMENT IN THE FIELD
}

\author{
BY
}

T/Major G. C. DANSEY-BROWNING, D.O.M.S., R.A.M.C.

THE basis of this article is the analysis of some 514 ophthalmic battle-casualties who were treated in a mobile ophthalmic unit during the Libyan Campaign.

The period covered ranged from the end of the year 1941 until the beginning of 1943. The analysis was compiled from notes made in the field and these were supplemented where possible by the results given on the follow-up cards received from the base hospitals.

\section{The Mobile Ophthalmic Unit}

The unit was usually attached to one of the casualty clearing stations of the most forward medical concentration area. As far as operations would permit all ophthalmic battle-casualties were routed to this casualty clearing station.

On account of the distances involved in the evacuation of the wounded, and the appalling nature of the desert tracks, thirty-six hours elapsed, on an average, between the time of the initial wound and the preliminary examination of the case in the ophthalmic unit. After the first field dressing was applied at the regimental aid post the sole treatment carried out during this stage of the evacuation was the oral administration of sulphonamides at the various field ambulances.

On arrival at the casualty clearing station the cases were " sorted" at the reception tent. The minor cases were sent direct to the vehicles of the ophthalmic unit, whilst major cases were sent to the "pre-operation" ward for treatment of shock and of their other injuries, and for X-ray investigation when necessary. The major cases were operated upon in the " theatre" of the casualty clearing station and usually remained in one of the wards for one or two days.

Thereafter, depending upon the severity of the injury, the case would be evacuated by-air, sea or road/rail to a base hospital. This journey might mean another three to four days before the case again came under the care of an ophthalmologist.

Nature of treatment in the Mobile Ophthalmic Unit.-It will readily be appreciated that, under the desert conditions outlined above; it was not possible to abserve the dictum of Würdemann " that field operations should be limited solely to the suture of lid-wounds, the removal of foreign bodies, and the use of antiseptics." 

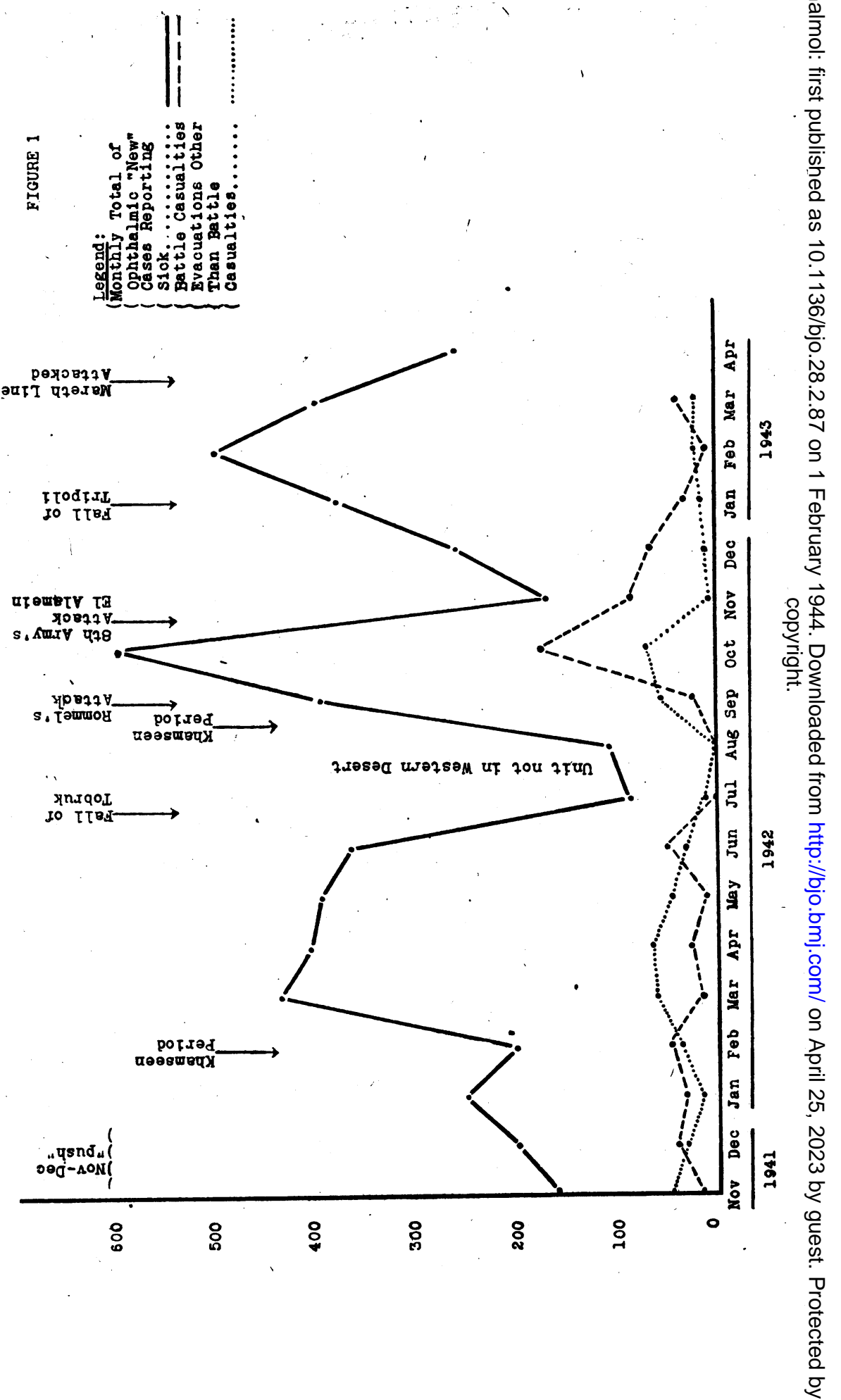
Thus it was found that although operative interference was confined to the absolute minimum, some two hundred " major" operations had to be performed.

Although the main clinical interest lies. in the treatment of the battle-casualty, it must not be forgotten that the main military value of the ophthalmic unit lies elsewhere. The prevention of the evacuation of cases of minor injury, or disease, or errors of refraction, from the forward areas to base is the unit's most important rôle. This point can be appreciated on studying the graph (Fig. 1), which shows the relative proportions of battle-casualties, ophthalmic " sick," and cases evacuated to base.

\section{Classification of Wounds}

Würdemann classified ophthalmic wounds as "penetrating," when the coats of the globe had been opened; "perforating," when the aqueous or vitreous had been tapped; and "doubleperforating," when there was a through-and-through wound of the globe.

In this report a single classification has been adopted-" penetrating," when the wound opened into the interior of the globe, and "perforating," when there was a through-and-through wound of the globe.

The wounds have been arranged uneler the headings of the weapon, projectile, etc., that caused them. The lesion reported is the most grave noted at the preliminary examination; other complications are indicated in the notes below.

The wounds in general.-Morax and Moreau describe the shellwound as the most common in the 1914-1918 casualties. These wounds still retain pride of place in the Libyan Campaign, with landmine-wounds running a good second.

The bullet-wound is still locally the most destructive; in 42.5 per cent. of such wounds, in this series, there was total disorganization of the globe.

The modern H.E. projectile has a particularly effective fragmentation. Thus though the wound of the eye is often small and self-sealed, it is but one of many wounds. The majority of the cases with these multiple injuries therefore require operation under general anaesthesia. Pentothal was found to be the best anaesthetic for use in the field.

Some types of wounds.-(1) Out of 67 cases of intra-ocular foreign body, 64 were demonstrated by X-ray. Two of the fragments were wooden, and the other one which was extremely small was visible with the ophthalmoscope, although the $\mathrm{X}$-ray was negative.

It was soon realized that accurate localization, by means, for example, of the ring-method, was not practicable under C.C.S. 
conditions. It was found, however, that with the ring laid on the cocainized limbus and a quick exposure (if the patient would remain steady), it was possible to differentiate with certainty between the intra-ocular and extra-ocular foreign body.

Some thirteen of the 67 cases with intra-ocular foreign body had the fragments removed by the giant-magnet, at base hospitals. Three to five applications of the magnet, at intervals of a few days, were often necessary before a positive result was obtained. Only one was removed in the field, i.e., at first application.

Removal by the posterior route, either through the original wound of entry or else through a scleral incision, appeared, in this series, to give less final upset of function.

This proportion of magnetisable foreign bodies is much larger than the modern use of steel-alloy led one to expect.

(2) Of the intra-orbital foreign bodies, some 22 fragments had perforated the globe, while some 31 had lodged in the orbit without penetrating the globe. Although these cases are listed under the F.B. section, their main complications were the result of concussion on the globe.

Michaelson has shown sections of cases, where double perforation of the globe has occurred, in which the repair of the exitwound has been accompanied by a proliferative choroiditis with the wound being plugged by a white, button-like, mass which projects forward into the vitreous.

(3) The statistics for the 1914-1918 war gave 6 per cent. of all casualties as due to indirect violence. In "this series 273 eyes have had to be listed under " effects of concussion," i.e., approximately half of the cases.

(a) Fracture of the bony orbit combined with extensive damage to the lids was present in 87 cases and was associated with total disorganization of the globe. The remains of the eye were removed as early as the patient's general condition would permit.

(b) "Traumatic keratitis" does not appear to have been granted its full clinical status. The concussion produces ruptures of Descemet's membrane. The aqueous may then find its way between the fibres of the substantia propria and produce opacification of the cornea. Owing to the force of the explosion the globe tends to roll upwards and the site of election is therefore the lower third of the cornea. All the cases noted here were slow 'of - resolution.

(c) Only two cases of detached retina were noted at the primary examination in the field, and only six more have been subsequently reported in this series.

Sulphonamide therapy.-The oral administration of sulphonamides during evacuation. was reinforced by the routine postoperative instillation of sterile sulphonamide ointment into the conjunctival sac. 
It is felt that this routine combined with the policy of minimal interference during the evacuation to the ophthalmologist was a distinct advance in combatting the problem of "hospital infection " in the field medical units.

Sympathetic Ophthalmitis.- "Many writers on the 1916-1918 casualties stressed the lessened incidence of sympathetic ophthalmitis and many warned against unnecessary enucleations." (Würdemann.) In 1918 the French authorities gave reports on only 39 cases of sympathetic ophthalmitis. In all of these operative interference appears to have been undertaken some considerable time (average 30 days) after the initial wound.

In the Middle East during the various campaigns it is understood that only one case (not in this series) of sympathetic ophthalmitis has been reported.

Severity of the Wounds.-Eighty-five of the five hundred and fourteen men had sustained wounds of the two eyes. In forty-five the wounds of both eyes were of grave prognosis; in fact, of these, 21 have been subsequently reported as " pensionably" blind. In the remaining forty the wound of one eye was of relatively minor import.

In addition to the 87 disorganized globes, 27 eyes had to be removed at base. Few men with major wounds of the eye were fit for front-line service again.

\section{Analysis of Cases (Reference Fig. 2)}

1. Intra-ocular Foreign Bodies. (67). Twenty-one of these were associated with prolapse of the iris, and five had prolapse of the vitreous. Therefore in the field 21 operations for abscission of prolapse and formation of a conjunctival hood had to be done. Nine cases had the sclera sutured, and simple conjunctival flaps had to be made for seven other wounds. One of these abscissions had to be repeated at base. In three more synechiae had to be divided, and the lens had to be removed in seven cases. Traumatic keratitis was a complicating factor in two cases, while three cases had air-bubbles in the anterior chamber. Thirteen cases developed traumatic cataract, the fragment being visible in the lens in eight of the cases. Lens matter appeared in the wound in six cases whilst the iris remained adherent to the wound edges in four. Vitreous came forward into the anterior chamber in one çase. Foreign bodies were visible on the fundus when examined with the ophthalmoscope in two cases, and were visible in the iris in another two.

Five cases developed panophthalmitis and in the large number of thirteen there was "cyclitis" reported at" the end of the first month. One case was reported to have " inter-retinal fluid oozing from the scleral wound "; in another " the vitreous haemorrhage 
became organized inferiorly" ; and in a third after a Haab+ve removal by the anterior route the anterior chamber did not reform.

Three men died from other wounds and 17 globes were enucleated.

The final reports on 14 were: vision $6 / 5$ one case, vision $6 / 9$ five cases, vision $6 / 12$ one case, vision $6 / 18$ one case, vision $6 / 36$ one case, vision with plus 10.00 D.S. 6/18 one case, and vision with plus 10.00 D.S. 6/60 four cases.

In another thirteen the results were: H.M. vision'four cases, " good P.L." five cases, " C.F.I. Metre " two cases, " No P.L." one case, and " recurrent hyphaema" in the other.

2. Perforating Intra-orbital Foreign Bodies. (22). Five of these were associated with prolapse of the iris and in another two there was vitreous prolapse. Of the foreign bodies removed by incision one was $\mathrm{Haab}+\mathrm{ve}$ and two Haab-ve.

In the field five operations for abscission of prolapse and formation of conjunctival hood were performed and the sclera was sutured in four cases. One operation for retinal detachment was done at a base hospital.

Complicating factors were : five cases with traumatic cataract with lens matter appearing in the wound in two cases. Subsequently two cases were reported to have retinal detachment ahd in one " the vitreous was full of blood.", Later two cases developed "cyclitis" and there was one case with posterior. synechiae. Other reports received were." iris pillars in the wound," " synchisis scintillans-one eye," "late macular changes" in another. "Large retinal haemorrhage, lowered tension and scarring over macula" -one case. Two eyes were

reported as with " faint P.L. being soft eyes with vascularization of the corneae." One case developed retinitis proliferans. 'Two eyes were enucleated.

For the results received as to the final function of the eyes : Vision $6 / 9$ one case; Vision $6 / 36$ one case ; Vision $6 / 60$ one case; Vision $2 / 60$ with field defect one case ; "C.F.I. metre" one case; "Vision H.M." one case; "P.L." six cases, and " blind eye" in three cases.

3. Non-penetrating Intra-òrbital Foreign Bodies. (31). As has been stated previously the main damage was caused by concussion. Two cases with associated skull injuries developed optic atrophy, while injury to the bony orbit produced another two cases of optic atrophy. 'Five had large lid injuries of which one required subsequent plastic repairs. Two of the orbits started to develop orbital cellulitis which fortunately resolved under oral sulphonamide therapy. Local damage to the muscle cone produced diplopia in one case. The final results of the cases with vitreous haemorrhages were varied. They ranged from "Vision 6/6; 
vitreous dust and retinal haemorrhages " to " good P.L., vitreous and macular haemorrhages and peripheral choroidal damage." Other reported results of these, were: "Organized blood in the vitreous, vision $6 / 36$ " ; "Vision $6 / 24$ " one case ; "Vision $2 / 60$ " one case ; "C.F.I. metre " one case ; "H.M." one case; "Good P.L." one case; "No P.L." one case, and " block of temporal retinal vessels " in another.

4. Conjunctival, Scleral and Corneal.Foreign Bodies. (192). These foreign bodies were as often sand as they were metallic and the corneal fragments gave, on the whole, surprisingly little upset of vision. Only the bigger pieces were removed in the field and then only if they were very irritating. The remainder tended to work their way out unaided. One case in particular had 62 pieces counted in his two eyes on arrival at base; his final vision was 6/24 right and left eyes.

Only two of the cases where foreign bodies were the main injury had a coincident keratitis, which supports the view that traumatic keratitis is a pure concussion effect.

One case only is reported to have developed a hypopyon ulcer, which necessitated section of the cornea with final vision of less than $6 / 60$.

The worst nebulae only affected vision to the extent of $6 / 36$ (two eyes), 6/12 (three eyes) and 6/18 (two eyes).

Five of the scleral foreign bodiès had small peripheral vitreous haemorrhages, and the effect on vision in the worst case was 6/24 and $6 / 12$. One case. with a shallow retinal detachment directly under the F.B. was missed at first examination. After operation at base for this detachment the final vision was less than $6 / 60$. One of the cases with a scleral foreign body developed a pyocyaneus abscess which, however, resolved fairly quickly and without ill-effects.

5. Disorganized globes. (87). Some 12 had fractures of the bones of the orbit, and 16 had gross damage to the lids. Nine of the cases had associated skull injuries. Plastic operations at base have been reported as necessary for 26 of these cases.

6. Lid Injuries. (46). Four of these have been reported to have needed plastic operation at base. Diplopia occurred in one case where the trochlea had been destroyed and in another case following fracture of the maxilla. One lacrymal sac fistula was reported in a case with a peripheral choroidal rupture and vision of $6 / 24$.

7. Traumatic keratitis. (7). As stated above these cases were slow of resolution, the length of time appearing to depend on the extent of the damage to Descemet's membrane. For example, two eyes slowly improved after two months but with vision reduced to $6 / 18$ and $6 / 60$. 
Although tears in Descemet's membrane have been noted as a result of war injury, "traumatic keratitis" does not appear to have been sufficiently widely recognised as a clinical entity.

8. Lens Injuries. Reports were received on only a few of these. One only had vision after operation corrected to $6 / 60$, two being less than 6/60, and one "Good P.L." Subluxation of one lens in one globe which was enucleated, showed a complete iridodialysis on pathological examination.

9. Vitreous Haemorrhages. (17). Resolution of the haemorrhages ofter revealed other lesions in the fundus, that had been obscured at the primary examination in the field. Thus two of the cases were reported with large retinal haemorrhages, one with a subhyaloid haemorrhage and one with macular changes.

The effect on final function was diverse and ranged from " vision 6/6 with medial opacities," 6/12 (two cases), 6/18 (one case), 6/36 (one case), " C.F.I. metre " (one case), " Good P.L." (two eases), down to "Organized blood in the posterior globe, blind eye " (one case). One eye was enucleated but the reasons for this have not been obtained.

10: Retinal detachment. (2). The results for these were disappointing as to function, being " organization of the subretinal haemorrhage with P.L. in lower field" and "P.L. with field defect."

11. Macular changes. One case with a "hole" had "good peripheral vision " ; this case was a released prisoner of war whose other eye had been enucleated whilst in enemy hands. One case with a macular haemorrhage had vision of $6 / 18$ after the first month, and another with " inferior retinal changes" had a " large upper field defect."

12. Rupture of the choroid. (3). The report on one case was " $6 / 36$ and two small retinal haemorrhages," and on another " 6/24 with cells in the retrolental space."

13. Ocular muscle palsy. (1). The muscle affected-was the superior rectus, but the main interest was that the damage resulting in the diplopia did not cause.any impairment of vision, which was $6 / 5$.

14. Ruptures of the globe without foreign bodies. In seventeen of these there was prolapse of the iris and in five prolapse of the vitreous. Six of these had traumatic cataract, four with lens matter presenting in the anterior chamber. Four cases developed cyclitis in the third and fourth weeks.

Seventeen abscissions 'of uveal tissue, and five scleral sutures were performed in the field. Two cases had lens extraction at base. One prolapse recurred and required further abscission at base but the anterior chamber did not reform and the result was "faint P.L." 
Five globes were enucleated at base, sections of the globes showing in one " retinal detachment" and in the other "panophthalmitis." A variety of reports on final function were received : " $6 / 5$ with iris still adherent to the wound" (one case); two cases with $6 / 6$; one case with peripheral choroidal rupture and vision of $6 / 6$; while two cases with the lens extracted were corrected to $6 / 9$ and $6 / 60$ respectively.

Vision was $6 / 24$ and $6 / 36$ in another two cases; one "less than 6/60" "; "Good P.L." (one case); " P.L. inferior field" (one case); and in yet another there was " temporary block of the retinal vessels."

\section{Conclusions}

If the "follow-up" received had been complete it would have been possible to assess the relative values of the field and base operations and to contrast the results obtained with those which are given by Würdemann for the casualties for 1916. In the notes above, the reports received have been summarised so that a general impression can be formed as to the number of eyes which retained useful function and those which were merely "kept."

The 1916 figures gave only 698 eyes as retained, out of two thousand ophthalmic casualties. The 27 globes " subsequently removed" and the 87 globes " totally disorganized" make 114 the number of eyes " lost" to date.

This figure, it is submitted, indicates that the modern policy? of forward ophthalmic treatment in the field has not had adverse ènd-results.

\section{Summary}

1. An analysis has been made of 514 ophthalmic casualties from the Libyan campaign who had been treated in a mobile ophthalmic unit.

2. The types of wounds encountered and their treatment have been outlined. Sixty-seven cases with intra-orbital foreign bodies were treated; fourteen of the foreign bodies were extracted by the giant magnet at base. Only one was extracted in the forward area.

Twenty-two of the intra-orbital foreign bodies had " perforated" the globe.

3. Owing to the terrain some two hundred men had to be operated on in the field unit.

4. It is suggested that "hospital infection" has been limited by the local' as well as oral administration of sulphonamides combined with a policy of " minimal interference" in the field medical units. No cases of sympathetic ophthalmitis have been reported from this series. 
5. Eighty-five of the cases had been wounds in both eyes. In forty-five the wounds of the two eyes were of grave prognosis, and in twenty-one the men have been subsequently reported as " blind."

6. Total disorganization of the globe occurred in eighty-seven cases, and the eyes were removed at the mobile unit. A further twenty-seven eyes have been reported to have been removed at base hospital.'

7. It is submitted that by general comparison with the statistics for the 1914-1918 war the results obtained for this campaign have not been adverse.

My especial thanks are due to 7262698, Pte. Baker, L., R.A.M.C., for his skilful and devoted nursing of the cases in the field, and to those of my colleagues who have made this article possible by sending back the "follow-up" cards.

\section{REFERENCES}

Injuries-WÜRDEMANN. " Ophthalmic Injuries."

Traumatic Keratitis-

Hudson. Trans. Ophthal. Soc. U.K., Vol. XLI, p. 254, 1920.

Juler. Proc. Roy. Soc. Med., Vol. XXVIII, p. 190, 1934.

Stallard. (Personal communication, 1943).

\section{ANNOTATION \\ The Value of an Optical Iridectomy}

It is common knowledge that in certain cases the performance of an iridectomy may be followed by great improvement in vision. Two cases bearing on this point stick in the writer's mind. In the first an old man from the workhouse came to out-patients with a blocked pupil, the synechia forming an almost complete ring. No red reflex could be obtained through the pupil, but the iris was not bombé, and perception of light was perfect. We thought it probable that the lens was largely opaque, but before deciding to try and extract it we took the man in for a preliminary iridectomy which was safely performed in the usual situation. It was not possible to remove a very large piece of iris and we had to be satisfied with a key hole result. When the bandage wds removed at the first dressing the patient announced that he could see well, and later examination established the fact that the lens in the area of iridectomy was completely clear. He got very good vision without having any more done.

The second case was a very old lady who was brought by her 